\title{
Prevenção da doença invasiva neonatal precoce pelo Streptococcus agalactiae: experiência de um hospital escola
}

\section{Preventing measures of early Streptococcus agalactiae invasive infections in newborns: experience of a school hospital}

\author{
Bruna Maria de Moraes Norcia ${ }^{1}$; Renata Aparecida Belei²; Uiara Rodrigues de \\ Oliveira Moraes ${ }^{3}$; Camilo Carvalho Reda ${ }^{4}$; Jaqueline Dario Capobiango ${ }^{5}$
}

\section{Resumo}

Streptococcus agalactiae ou Streptococcus do grupo B (SGB) é um importante patógeno responsável por infecções bacterianas invasivas em neonatos, como a sepse, pneumonia e meningite. O objetivo deste estudo foi avaliar a adesão as medidas de prevenção de infecção neonatal precoce pelo SGB, conforme recomendado pelo Centers for Disease Control and Prevention (CDC), em dois períodos, antes e após o envolvimento da Comissão de Controle de Infecção Hospitalar (CCIH) como uma rotina do serviço e avaliar se houve impacto na morbidade e mortalidade neonatal no segundo período analisado. Tratase de um estudo de coorte, de caráter retrospectivo, realizado no Hospital Universitário de Londrina (HU), PR. Foram analisadas 800 fichas da CCIH de parturientes, 107 do período de 2008 a 2009 e 693 do período de 2011 a 2012. Após envolvimento da CCIH na implantação do protocolo do CDC, houve incremento de $88,2 \%$ na coleta de swabs vaginal e retal das parturientes, aumento de $95,7 \%$ de realização de uroculturas e aumento de $86,4 \%$ na realização de antibioticoprofilaxia intraparto. Em relação aos recém-nascidos sintomáticos, foram diagnosticados 7/107 (6,5\%) no período de 2008 a 2009 e 49/693 (7,0\%) no período de 2011 a 2012. Recém-nascidos sintomáticos evoluíram para óbito: 1/107 (0,9\%) no período de 2008 a 2009 e $12(1,7 \%)$ no período de 2011 a 2012 . Concluiu-se que o envolvimento da CCIH na implantação do protocolo do CDC, foi fundamental na obtenção de uma maior adesão às medidas de profilaxia de doença invasiva precoce pelo SGB, porém não houve impacto na morbidade e mortalidade neonatal.

Palavras-chave: Streptococcus agalactiae. Recém-nascido. Gestantes. Infecção.

\begin{abstract}
Streptococcus agalactiae, or Streptococcus group B (GBS), is an important pathogen responsible for invasive bacterial infections in newborns like sepsis, pneumonia and meningitis. It is aimed in this study to assess adherence to the preventing measures of early neonatal infection by GBS as recommended by Centers for Disease Control and Prevention (CDC) in two periods, before and after the involvement of the hospital infection control committee (HICC) and to verify whether there was impact in neonatal morbidity and mortality in the second analyzed period. A cohort retrospective study was performed at the University Hospital of Londrina, PR-Brazil. Records of HICC of 800 mothers, 107 and 693, in the periods 2008-2009 and 2011-2012, respectively. After HICC involvement in the implementation

\footnotetext{
${ }^{1}$ Residente de Infectologia Pediátrica da Universidade Estadual de Londrina Email: brunanorcia@hotmail.com

${ }^{2}$ Doutora em Educação Área de Ensino na Educação Brasileira. Enfermeira da Comissão de Controle de Infecção Hospitalar do Hospital Universitário da Universidade Estadual de Londrina. Email: rabellei@yahoo.com.br

${ }^{3}$ Acadêmica do Curso de Enfermagem da Universidade Estadual de Londrina. Email: uimoraes@hotmail.com

${ }^{4}$ Acadêmico do Curso de Medicina da Universidade Estadual de Londrina. Email: ueucamilo@yahoo.com.br

${ }^{5}$ Docente do Departamento de Clínica Médica da Universidade Estadual de Londrina. Email: jaquede@uel.br
} 
of the CDC protocol, there was an increase of $88.2 \%$ in the collection of vaginal and rectal swabs of parturients, an increase of $95.7 \%$ in urine culture and an increase of $86.4 \%$ in intrapartum antibiotic prophylaxis. Regarding symptomatic neonates, there were diagnosed 7/107 (6.5\%) in the period from 2008 to 2009 and $49 / 693$ (7.0\%) in the period 2011 to 2012. In relation to symptomatic newborns, $1 / 107(0.9 \%)$ died in the period 2008-2009 and 12/693 (1.7\%) in the period 2011-2012. We concluded that the involvement of the HICC in the CDC protocol implementation was fundamental to get greater adherence to measures of early prophylaxis of invasive disease by GBS, but there was no impact on neonatal morbidity and mortality.

Keywords: Streptococcus agalactiae. Newborn. Pregnant women. Infection.

\section{Introdução}

Na década de 1970, Streptococcus agalactiae, também referido como estreptococo do grupo B (SGB), um diplococo gram-positivo, emergiu como importante patógeno causador de infecções bacterianas invasivas, como sepse, pneumonia e meningite em neonatos $(\mathrm{RN})$, principalmente na primeira semana de vida (COSTA; BRITO, 2011; BERALDO et al., 2004). Desde então, se mantém como principal agente infeccioso responsável pelas elevadas taxas de morbidade e mortalidade nos países industrializados, acometendo 0,5 a 3 neonatos em 1000 nascidos vivos (BORGER et al., 2005; POGERE et al., 2005; GRASSI; DINIZ; VAZ, 2001). O SGB também pode ser causa de infecção na gestante (15\%), o que ocasiona várias complicações como corioamnionite, endometrite, infecção do trato urinário e de sítio cirúrgico (MELIN, 2011; CDC, 2010).

A infecção sistêmica pelo SGB apresenta duas formas de manifestação clínica no período neonatal, infecção de início precoce e infecção de início tardio. A infecção de início precoce ocorre nos primeiros sete dias de vida, em $2 / 3$ dos casos manifesta-se nas primeiras 24 horas de vida (PINHATA, 2010; SILVA; RITCHMANN, 2006). As formas clínicas de manifestação precoce mais comuns são sepse, bacteremia sem localização da infecção, pneumonia e meningite (MELIN; 2011; TEIXEIRA et al., 2005; ROCHA et al., 1999). A infecção de início tardio ocorre em $1 / 3$ dos casos e manifesta-se entre sete dias e 12 semanas de vida. Neste caso, pode ser consequência de transmissão horizontal ou nosocomial, raramente vertical. Apresenta-se como meningite, doença localizada em tecidos moles, ossos e articulações (GRASSI; DINIZ; VAZ, 2001; BENITZ; GOULD; DRUZIN, 1999; AMARAL, 2008).

O fator determinante para a infecção neonatal precoce pelo SGB parece ser sua presença no trato genital materno ao nascimento. $\mathrm{O}$ terço distal da mucosa vaginal é colonizado a partir do canal ano-retal, por isso estes são os locais indicados para a pesquisa do SGB. O aparelho urinário também é um importante sítio de infecção por essa bactéria, especialmente durante a gravidez, quando usualmente manifesta-se como bacteriúria assintomática (SCHRAG; WHITNEY; SCHUCHAT, 2000; ARRIERO; RICHTMANN; BALTIER, 2008).

O risco para o RN adquirir infecção através da transmissão vertical está diretamente relacionado ao número absoluto de micro-organismos presentes no canal de parto ao nascimento e ausência de anticorpos específicos contra o polissacarídeo capsular do SGB que são transferidos da mãe para o RN nas últimas 10 semanas de gestação. Dessa forma, o RN de mãe colonizada, principalmente o pré-termo, é 10 a 15 vezes mais susceptível a desenvolver a doença invasiva precoce (COSTA; BRITO, 2011). Os fatores de risco mais importantes para infecção neonatal precoce incluem: história prévia de irmão com doença invasiva por SGB, bacteriúria por SGB durante a gestação, trabalho de parto com idade gestacional inferior a 37 semanas, ruptura de membranas igual ou superior a 18 horas 
e temperatura intraparto maior ou igual a $38^{\circ} \mathrm{C}$. (PINHATA, 2010; TEIXEIRA et al., 2005; APGAR; GREENBERG; YEN, 2005).

Uma estratégia de prevenção da doença neonatal precoce é obter o diagnóstico das gestantes colonizadas por SGB, com a realização da cultura de rotina para pesquisa do SGB em toda gestante, entre a $35^{\mathrm{a}}$ e $37^{\mathrm{a}}$ semanas de idade gestacional (IG). O material obtido por meio de swab deve ser colhido do terço distal da vagina e anorretal. A quimioprofilaxia com antimicrobianos em gestantes colonizadas por SGB deve ser feita intraparto, logo após o início do trabalho de parto ou ruptura das membranas, sendo necessárias pelo menos duas doses de antibióticos (penicilina ou ampicilina) com intervalo de quatro horas, antes da resolução do parto, para que uma menor porcentagem de RN se torne colonizada e infectada pelo SGB ao nascimento. A quimioprofilaxia é hoje a melhor arma no combate à doença invasiva pelo SGB (CDC, 2010; MELIN, 2011).

Segundo de Cueto e colaboradores (1998), a taxa de transmissão do SGB em mães que receberam penicilina cristalina por período superior a quatro horas antes do parto, a taxa de transmissão foi de $1,2 \%(p<0,005)$, enquanto que para gestantes que receberam penicilina entre uma e duas horas antes do parto foi de $28 \%$ e as que receberam por período menor que uma hora antes do parto a taxa foi de $46 \%$.

A partir de 2007, foi divulgado o protocolo do Centers for Disease Control and Prevention (CDC) sobre a prevenção de doença invasiva neonatal precoce pelo EGB em nosso serviço. As coletas de swab vaginal e retal das gestantes foram estabelecidas como rotina do setor de ginecologia e obstetrícia do Ambulatório de Especialidades do Hospital Universitário de Londrina, em 2007. Em 2010, foi realizada nova divulgação das recomendações do CDC e a Comissão de Controle de Infecção Hospitalar (CCIH) do Hospital Universitário de Londrina assumiu a vigilância dos casos suspeitos e confirmados de gestantes portadoras de SGB. Desde então, foram realizados registros nos prontuários das mulheres internadas na maternidade deste hospital portadoras de SGB ou com fatores de risco para o SGB, para que a equipe médica pudesse realizar a antibioticoprofilaxia. A equipe da $\mathrm{CCIH}$ também passou a recomendar a realização de cultura de $s w a b$ nas mulheres que ainda não haviam realizado este exame. Em 2011 foi implantada também, a rotina de coleta de swab vaginal e retal das gestantes nas unidades básicas de saúde do município de Londrina.

O presente estudo foi realizado com o objetivo de avaliar a importância do envolvimento da Comissão de Controle de Infecção Hospitalar o Hospital Universitário de Londrina na adesão às medidas preconizadas pelo CDC para prevenção da doença invasiva neonatal precoce bem como o impacto da adesão a essas medidas na morbidade e mortalidade neonatal.

\section{Metodologia}

Trata-se de um estudo de coorte de caráter retrospectivo, realizado no Hospital Universitário de Londrina, PR - Brasil. A população em estudo foi constituída de parturientes da maternidade deste hospital e seus recém-nascidos. Foram analisados os dados de dois períodos: de 01/03/2008 a 30/04/2009, início da implantação do protocolo do CDC em nosso serviço e de 01/03/2011 a 30/04/12 após envolvimento da $\mathrm{CCIH}$, que assumiu a vigilância dos casos suspeitos e confirmados de gestantes portadoras de SGB como uma rotina de seu serviço.

A coleta de dados foi realizada com ficha específica da $\mathrm{CCIH}$, que contém o registro das parturientes deste hospital e seus recém-nascidos, com informações sobre fatores de risco, culturas de swab e urina, histórico do arto, realização de antibioticoprofilaxia e evolução do RN. Dados referentes à frequência de nascidos vivos foram obtidos do setor de estatística do HU. Os dados 
foram tabulados e analisados pelo programa Epi Info, versão 3.4.3 de novembro de 2007 e GraphPad Prism versão 5.0 de 2007.

O presente estudo foi aprovado pelo Comitê de Ética em Pesquisa envolvendo Seres Humanos da Universidade Estadual de Londrina, parecer $n^{\circ}$. 134/2012.

\section{Resultados}

Foram analisadas 800 fichas da $\mathrm{CCIH}$ de parturientes do HU, 107 do período de 2008 a 2009 e 693 do período de 2011 a 2012. No período de 2008 a 2009, houve 972 nascidos vivos na maternidade do HU, portanto 11\% (107/972) de parturientes analisadas. No período de 2011 a 2012, houve 1.126 nascidos vivos na maternidade do HU, portanto $61 \%$ (693/1126) de parturientes analisadas. Houve incremento de $82 \%$ de gestantes analisadas.

Foi realizada coleta de swab vaginal e retal no pré-natal em $9 / 107$ parturientes $(8,4 \%)$ no período de 2008 a 2009 e em 496/693 (71\%) no período de 2011 a 2012, com aumento de 88,2\% de realização de coleta de $s w a b$ (Figura 1). Desses swabs vaginal e retal coletados em 2008 a 2009, 3/107 (2,8\%) foram positivos e no período de 2011 a 2012, 44/693 $(6,3 \%)$, com elevação na positividade das amostras em 55,6\% (Gráfico 1).

Gráfico 1- Frequência de realização e de positividade de swabs vaginal e retal para pesquisa de SGB em gestantes em dois períodos: 107 amostras analisadas em 2008-2009 e 693 amostras analisadas em 20112012, em Londrina/PR.

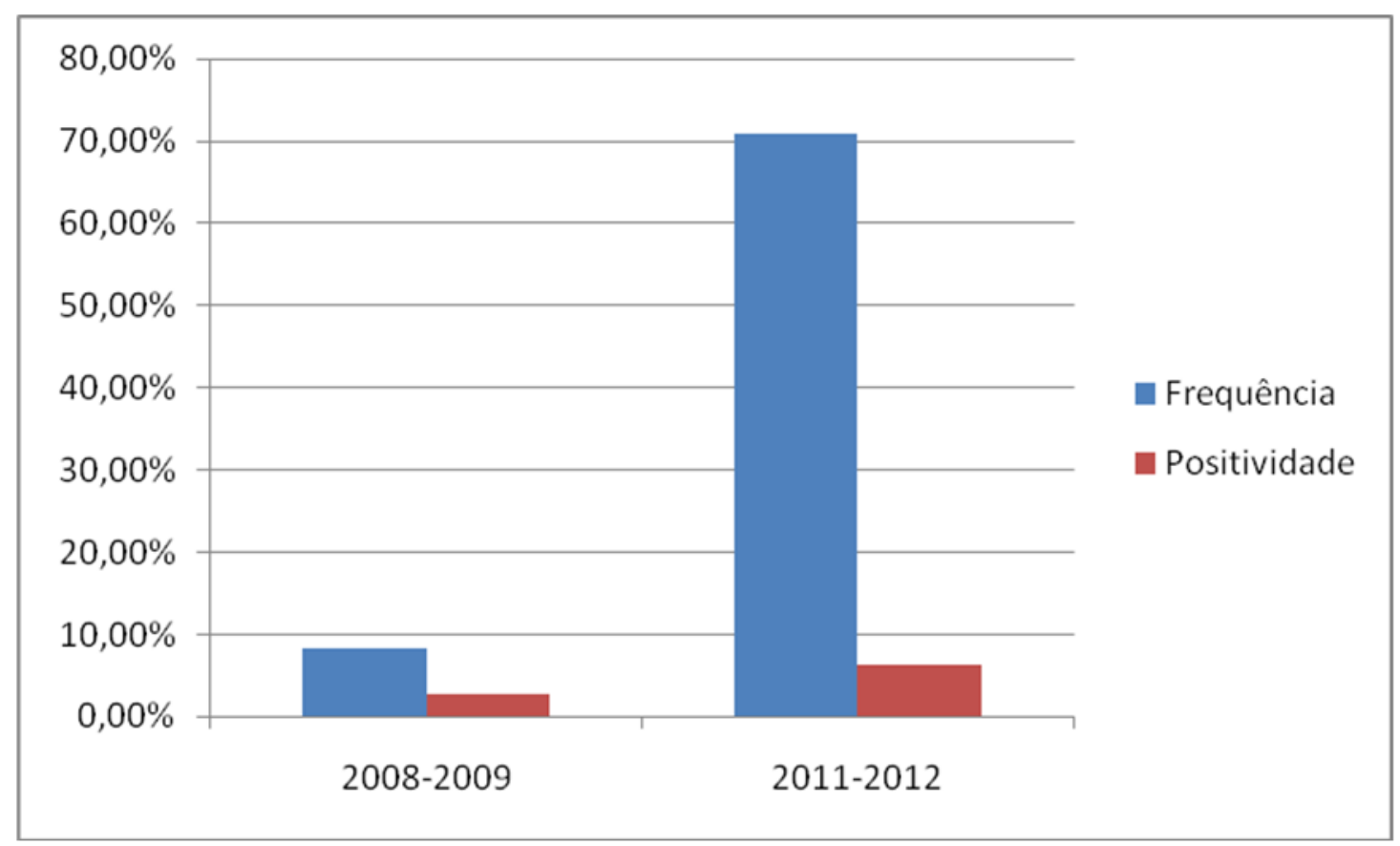

Fonte: autor

Houve realização de urocultura em 4/107 parturientes $(3,7 \%)$ no período de 2008 a 2009 e 592/693 (85,4\%) no período de 2011 a 2012, com aumento de 95,7\% de realização de uroculturas (Figura 2). Entre as amostras de urocultura com crescimento de SGB, 1/107 (0,9\%) foi positiva no período de 2008 a 2009 e 14/693 (2\%) no período de 2011 a 2012, houve incremento de $55 \%$ na positividade das uroculturas. (Gráfico 2) 
Gráfico 2 - Frequência de realização e de positividade de uroculturas para pesquisa de SGB em gestantes em dois períodos: 107 amostras analisadas em 2008-2009 e 693 amostras analisadas em 2011-2012, Londrina/ PR.

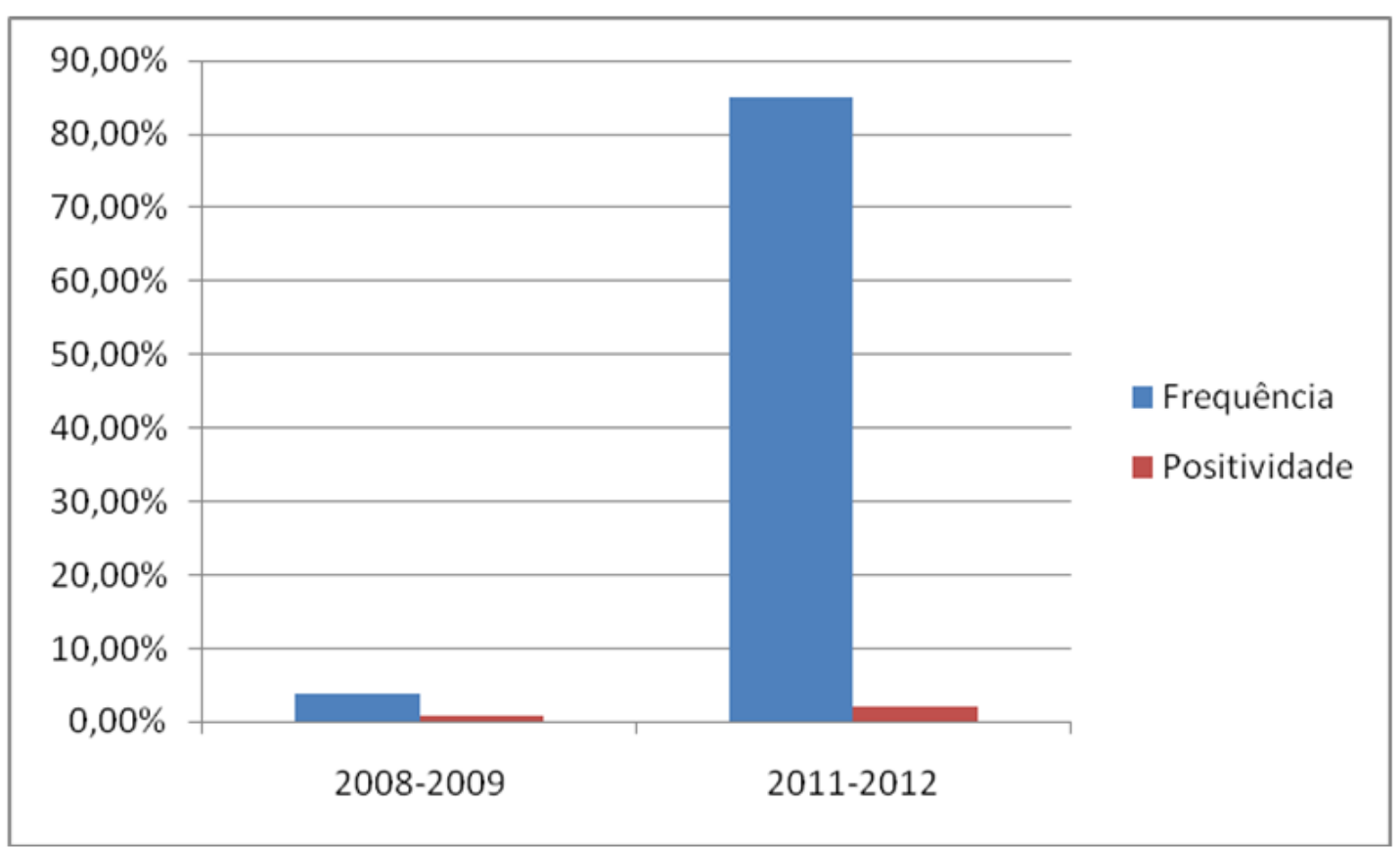

Fonte: autor

A antibioticoprofilaxia intraparto (AIP), 2009 e 49/693 (7,0\%) no período de 2011 a 2012 , foi realizada em $4 / 107(3,8 \%)$ no período de com incremento de $15,2 \%$ de pacientes sintomáticos, 2008 a 2009 e $51(27,9 \%)$ no período de 2011 porém não houve diferença significativa entre os a 2012, com elevação de $86,4 \%$ de realização de antibioticoprofilaxia.

Em relação aos RN sintomáticos, foram diagnosticados $7 / 107$ (6,5\%) no período de 2008 a dois períodos $(\rho=0,6874$; IC95\% $0.3478-2.007)$. As principais topografias das infecções apresentadas pelos RNs nos dois períodos analisados estão descritas na tabela 1 . 
Tabela 1- Principais topografias das infecções encontradas nos recém-nascidos nos dois períodos analisados: 7 infecções em 107 RN em 2008-2009 e 49 infecções em 693 RN em 2011-2012, Londrina/PR.

\begin{tabular}{lcccl}
\hline $\begin{array}{l}\text { TOPOGRAFIA DAS } \\
\text { INFECÇÕES }\end{array}$ & $2008-2009$ & $2011-2012$ & \multicolumn{1}{c}{$\mathrm{p}^{*}$} & OR (IC 95\%) \\
\hline SEPSE & $2 / 107(1,8 \%)$ & $21 / 693(3,0 \%)$ & 0,7567 & $0,6095(0,1408-2,638)$ \\
MENINGITE & $0 / 107(0,0 \%)$ & $11 / 693(1,6 \%)$ & 0,3764 & $0,2760(0,0161-4,722)$ \\
PNEUMONIA & $5 / 107(4,6 \%)$ & $10 / 693(1,4 \%)$ & 0,0911 & $2,8160(0,8662-9,158)$ \\
ITU & $0 / 107(0,0 \%)$ & $4 / 693(0,6 \%)$ & 1,0000 & $0,7127(0,0380-13,34)$ \\
OUTROS & $1 / 107(0,9 \%)$ & $3 / 693(0,4 \%)$ & 0,4376 & $2,1700(0,2235-21,06)$ \\
\hline TOTAL & $7 / 107(6,5 \%)$ & $49 / 693(7,0 \%)$ & 1,0000 & $0,9220(0,4053-2,088)$ \\
* Teste exato de Fisher & & & \\
Fonte: autor & & & &
\end{tabular}

Recém-nascidos sintomáticos evoluíram para óbito: 1/107 (0,9\%) no período de 2008 a 2009 e $12(1,7 \%)$ no período de 2011 a 2012, não houve diferença significativa entre os dois períodos $(\rho=1,0000$; IC95\% 0,06887-4,162).

\section{Discussão}

No Brasil, a colonização anal e/ou vaginal pelo EGB encontrada na população de gestantes varia de 15 a 25\% (MOCELIN et al., 1995; BERALDO et al., 2004; POGERE et al., 2005), semelhante a encontrada nos EUA, cuja colonização das gestantes é de aproximadamente 20\% (SCHRAG; WHITNEY; SCHUCHAT, 2000; MELIN, 2011). Em nosso serviço não pudemos considerar a prevalência de colonização, pois houve uma perda importante de casos 376/693 (52\%). Porém, obtivemos uma maior detecção de gestantes com EGB no $2^{\circ}$ período analisado, tanto para as amostras de swab quanto para as uroculturas. van Dyke e colaboradores (2009) também avaliaram duas coortes retrospectivas no período de 1998 a 1999 e no período de 2003 a 2004, totalizando 254 RNs portadores de SGB e 7.437 RNs não portadores de SGB. A taxa de screening no $1^{\circ}$ período foi de $48 \%$ e no $2^{\circ}$ período foi de $85 \%$. Foi realizada quimioprofilaxia intraparto em $87 \%$ recém-nascidos de termo (RNT) e 63\% em recém-nascidos pré-termo (RNPT), no $2^{\circ}$ período. Em 13,4\% das mulheres não foi realizado screening pré-natal.

A eficácia da quimioprofilaxia intraparto é de 80\% (SMAILL, 2009). Das gestantes colonizadas analisadas em uma maternidadeprivada de São Paulo, $28 \%$ receberam antibioticoprofilaxia intraparto adequadamente (ARRIERO; RITCHMANN; BALTIERI, 2008). Dados semelhantes foram encontrados em nosso serviço, após o envolvimento da CCIH na vigilância dos casos suspeitos e confirmados de gestantes portadoras de EGB. Há risco de reação materna à penicilina, sendo que de 7 a $10 \%$ das reações evolui de forma leve e ocorre uma reação fatal a cada 50.000 a 100.000 doses aplicadas (CDC, 2010). Apesar do baixo risco, há preocupação a cerca dos efeitos colaterais da penicilina cristalina intraparto, como anafilaxia e morte materna, e aumento de bactérias gram negativas resistentes nos RN. Embora os estudos sejam controversos a este respeito, a magnitude dessas preocupações não é suficiente para superar os benefícios da IAP na prevenção da sepse neonatal precoce (MELIN, 2011). Em nosso serviço, nenhuma parturiente apresentou reação adversa à administração de penicilina cristalina endovenosa.

Outra preocupação é o aumento da resistência aos antibióticos usados para tratamento da sepse precoce após a introdução da AIP (RAUSCH et al., 2008). Há relato de aumento de sepse precoce por bactérias resistentes, além de maior frequência 
de enterobactérias nos recém-nascidos pré-termo, de baixo peso, ou muito baixo peso, cujas mães receberam AIP (MOORE; SCHRAG, SCHUCHAT, 2003).

Cerca de 30 a $70 \%$ dos nascidos de mãe colonizadas por EGB se tornam colonizados temporariamente. A maioria permanece assintomática, porém 1 a $3 \%$ desenvolve doença severa (MELIN, 2011). Em nosso serviço apesar de ter havido uma perda de registro de 376 casos no $1^{\circ}$ período, não foi o observado redução dos casos de doença do RN no segundo período analisado.

A mortalidade por sepse neonatal precoce (SNP) declinou nas últimas décadas, no mundo. Em 1970 acometia 55\% dos neonatos, na década de 80 acometia de 10 a $15 \%$ dos recém-nascidos e recentemente acomete de 3 a $5 \%$ dos neonatos (SCHRAG; WHITNEY; SCHUCHAT, 2000). Em três ensaios clínicos com 852 mulheres foram avaliados dois grupos: antibioticoprofilaxia intraparto versus sem tratamento. O uso da AIP não reduziu significativamente a mortalidade por EGB e por outras causas, apesar de ter ocorrido diminuição da incidência de sepse precoce por EGB (RR: 0,17 IC 95\% 0,04-0,74) (OHLSSON; SHAH; VIBHUTI, 2009). À semelhança da literatura, a mortalidade neonatal encontrada foi baixa, porém não reduziu no $2^{\circ}$ período analisado.

\section{Conclusão}

O presente estudo demonstra a importância do envolvimento da CCIH na implantação do protocolo do CDC e na obtenção de maior adesão às medidas de profilaxia de doença invasiva precoce neonatal pelo Streptococcus do grupo B no Hospital Universitário de Londrina, porém não houve impacto na morbidade e mortalidade neonatal.

\section{REFERÊNCIAS}

AMARAL, E.; Estreptococo do grupo B: rastrear ou não rastrear no Brasil? Eis a questão. Revista Brasileira de Ginecologia e Obstetrícia, Rio de Janeiro, v.30, n.6, p.274-280, jun. 2008.

ARRIERO, G.D.; RICHTMANN, R.; BALTIERI, S.R. Impacto do protocolo de prevenção de sepse neonatal pelo Estreptococo do grupo B. Pro Matre Paulista, São Paulo, p.1-13, 2008.

APGAR, B.S.; GREENBERG, G.; YEN, G. Prevention of group B streptococcal disease in the newborn. American Family Physician, v.71, n.5, p.903-910, mar.2005.

BENITZ, W.E.; GOULD, J.B.; DRUZIN, M.L. Antimicrobial prevention of early-onset group B Streptococcal sepsis: estimates of risk reduction based on a critical literature review. Journal of the American Academy of Pediatrics, Illinois, v.103, n.6, p.1145-1149, jun.1999.

BERALDO, A.; BRITO, S.J.; SARIDALKS, H.O; MATSUO, T. Prevalência da colonização vaginal e anorretal por Estreptococo do grupo B em gestantes do terceiro trimestre. Revista Brasileira de Ginecologia e Obstetrícia, Rio de Janeiro, v.26, n.7, p.543-549, jul. 2004.

BORGER, I.L.; OLIVEIRA, R.E.C.; CASTRO, A.C.D.; MONDINO, S.S.B. Streptococcus agalactiae em gestantes: prevalência de colonização e avaliação da susceptibilidade aos antimicrobianos. Revista Brasileira de Ginecologia e Obstetricia, Rio de Janeiro, v.27, n.10, p.575-579, out. 2005.

CDC. Provisional Recommendations for the Prevention of Perinatal Group B Streptococcal Disease. Date of posting of provisional recommendations: July 29, 2010 disponível em $<$ http://www.cdc.gov/abcs/reports. $>$. Acesso em: jun 2012. 
COSTA, H.P.F.; BRITO, A.S. Prevenção $d a$ doença perinatal pelo estreptococo do grupo $B$. Sociedade Brasileira de Pediatria- Educação médica continuada. 2011. Disponível em:< http://www. sbp .com.br/ pdfs/ SBPEG BCDC 2011-(2).pdf >. Acesso em: 2 mai. 2012.

DE CUETO, M.; SANCHEZ, M.J.; SAMPEDRO, A.; MIRANDA, J.A.; HERRUZO, A.J.; ROSAFRAILE, M. Timing of intrapartum ampicillin and prevention of vertical transmission of group B streptococcus. Obstetrics \& Gynecology, Granada, v.91, n. p.112-114, jan. 1998.

GRASSI, M.S.; DINIZ, E.M.A.; VAZ, F.A.C. Métodos laboratoriais para diagnóstico da infecção neonatal precoce pelo Streptococcus beta hemolítico do grupo B. Pediatria, São Paulo, v.23, n.3, p.232240, set. 2001.

MELIN, P. Neonatal group B streptococcal disease: from pathogenesis to preventive strategies. European Journal of Clinical Microbiology \& Infectious Diseases Clinical, Berlim, v.17, p.12941303, mai. 2011.

MOCELIN, C.O.; CARVALHO, D.A.F.; BRITES, C.; CHRISTOFOLLI, D.; MOCELIN, A.O.; FRACALANZA,S.E.; et al. Isolamento de Streptococcus agalactiae de gestantes na Regiäo de Londrina-PR. Revista Brasileira de Ginecologia e Obstetrícia, Rio de Janeiro, v.17, n.9, p. 915-918, out. 1995.

MOORE, M.R.; SCHRAG, S.J.; SCHUCHAT, A. Effects of intrapartum antimicrobial prophylaxis for prevention of group-B-streptococcal disease on the incidence and ecology of early-onset neonatal sepsis. The Lancet Infectious Disease, v.3, n.4, p.201-213, abr. 2003.

OHLSSON A., SHAH VIBHUTI S. Intrapartum antibiotics for known maternal Group B streptococcal colonization. Cochrane Database of Systematic Reviews. The Cochrane Library, v. 1, n. CD007467. DOI: 10.1002/14651858.CD007467. pub3, jul. 2009.
PINHATA, M.M.M. Infecção por streptococcus B: prevenção e conduta. Programa de Atualização em Neonatologia PRORN, Porto Alegre, Arthemed, ciclo 9, v.3, p.41-85, 2010.

POGERE, A.; ZOCOLLI, C.M.; TOBOUTI N.R.; FREITAS, P.F.; D'ACAMPORA, A.J.; ZUNINO, J.N. Prevalência da colonização pelo estreptococo do grupo $\mathrm{B}$ em gestantes atendidas em ambulatório de pré-natal. Revista Brasileira de Ginecologia e Obstetricia, Rio de Janeiro, v.27, n.4, p.174-180, jul. 2005.

RAUSCH, A.V.; GROSS, A.; DROZ, S.; BODMER, T.; SURBEK, D. Group B Streptococcus colonization in pregnancy: prevalence and prevention strategies of neonatal sepsis. Journal Perinatal Medicine, v.37, n.2, p. 124-129, out. 2008.

ROCHA, J.L.S.; DUARTE, G.; CUNHA, S.P.; NOGUEIRA, A. A.; MAUAD, F.F. Antibioticoprofilaxia com ampicilina na rotura prematura das membranas. Estudo randomizado e duplo cego. Revista Brasileira de Ginecologia e Obstetrícia, Rio de Janeiro, v.21, n.5, p.251-258, jul. 1999.

SCHRAG, S.J.; WHITNEY; C.G.; SCHUCHAT, A. Neonatal group B streptococcal disease: how infection control team can contribute to prevention efforts. Infection Control and Hospital Epidemiology, Chicago, v.21, n.7,p. 473-483, jul.2000.

SILVA, L. J.; RITCHMANN, R. Vacinas em desenvolvimento: estreptococo do grupo $\mathrm{B}$, herpeszóster, HIV, malária e dengue. Jornal de Pediatria, Porto Alegre, v.82, p.115-123, mai/jun. 2006.

SMAILL, F. M. Intrapartum antibiotics for Group B streptococcal colonisation. Cochrane Database of Systematic Reviews. The Cochrane Library, v. 1, n. CD000115. DOI: 10.1002/14651858.CD000115, out. 2009. 
TEIXEIRA, M.G.J; ISSA, A.; MAGALHÃES, C.K.; SOARES, A. J. S.; SAMPAIO, F.B.; ALVES W.A.; et al. Endocardite aguda por Streptococcus agalactiae: relato de caso. Revista da Sociedade de Cardiologia do Estado do Rio de Janeiro, Rio de Janeiro, v.18, p.477-479, set/out. 2005.

VAN DYKE, M.K.; PHARES, C.R.; LYNFIELD, R.; THOMAS, A.R.; ARNOLD, K.E; CRAIG, A.S.; at al. Evaluation of universal antenatal screening of group B streptococcus. The New England Journal of Medicine, Massachussetts, v.360, n.25, p.26262636, jun. 2009.

Recebido em: 17 abr. 2013

Aceito em: 23 jun. 2014 
